\title{
Decoding the ECD Spectra of Poly(phenylacetylene)s: Structural Significance
}

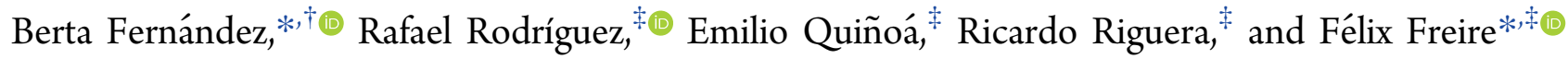 \\ ${ }^{\dagger}$ Department of Physical Chemistry, University of Santiago de Compostela, 15782 Santiago de Compostela, Spain \\ ‡Centro Singular de Investigación en Química Biolóxica e Materiais Moleculares (CiQUS) and Departamento de Química Orgánica, \\ Universidade de Santiago de Compostela, 15782 Santiago de Compostela, Spain
}

Supporting Information

ABSTRACT: The role of the main dihedral angles in the electronic circular dichroism (ECD) spectra of poly(phenylacetylene)s (PPAs) was estimated by using timedependent density functional theory (TD-DFT) for oligo(phenylacetylene)s $(n=12)$. These studies reveal that in cistransoidal arrangements, the first Cotton effect is dominated by excitations involving molecular orbitals (MOs) mainly related to the polyene backbone. Hence, for this scaffold, the \pm sign of the first Cotton effect reflects the $\mathrm{P} / \mathrm{M}$ helical sense of the internal helix of the polymer. However, in cis-cisoidal arrangements, contribution of MOs in the polyene and the aryl rings of the PPA backbone produce the first Cotton effect
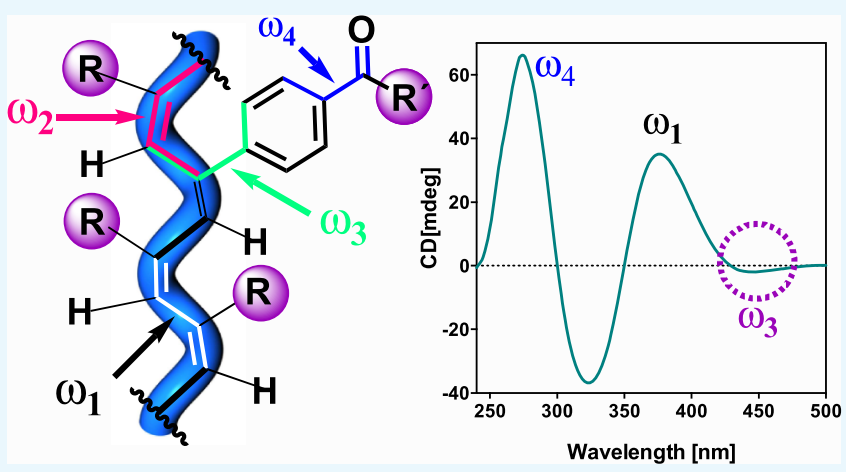
band. As a result, two different ECD signatures with three or four alternating Cotton effects can be produced depending on the sign of the $\omega_{1}$ and $\omega_{3}$ dihedral angles which determine the helical sense of the polyene $\left(\omega_{1}\right)$ and the relative orientation of the aryl ring toward the polyene $\left(\omega_{3}\right)$, respectively. Thus, on the one hand, if $\omega_{1}$ and $\omega_{3}$ rotate in opposite directions, a CD with three alternating Cotton effects is observed, where the sign of first Cotton correlates with the $\mathrm{P} / \mathrm{M}$ helical sense of the polyene. On the other hand, if $\omega_{1}$ and $\omega_{3}$ rotate in the same direction, a $\mathrm{CD}$ signature with four alternating Cotton effects is produced where the information relative to the $\mathrm{P} / \mathrm{M}$ helical sense of the polyene is contained in the second Cotton effect.

\section{INTRODUCTION}

To develop novel functional materials based on helical polymers, it is necessary to identify the different parameters involved in polymer folding. Thus, the elucidation of the secondary structure in some helical polymers such as poly(phenylacetylene)s (PPAs) ${ }^{1-5}$ is still a challenge because of the difficulty in obtaining information about the different helical parameters, that is, helical pitch and helical sense. ${ }^{6}$

During the last decade, great effort has been made combining information from different structural techniques to finally get a good approximation of the helical structure adopted by these polymers. However, this is a difficult task because of the intrinsic properties of the polymers, where the same fragment is repeated along the polymer chain (monomer repeating unit) (Figure 1a). Moreover, PPAs form two coaxial helices, one described by the polyene backbone (internal helix) and the other one described by the pendants (external helix), which can rotate in the same or opposite directions depending on the dihedral angle between conjugated double bonds (Figure 1b).,7

In PPAs, the internal helix is not stabilized by any supramolecular interaction within the polyene backbone. Thus, the adoption by the polymer of a certain helical structure responds to the conformational composition at the pendant groups and to the presence of either supramolecular or steric interactions among pendants.

Nevertheless, considering the flexibility of the polyene skeleton, these interactions may originate many different structures depending on the dihedral angle between conjugated double bonds $\left(\omega_{1}\right)$ (Figure 1a). Thus, on the one hand, if this dihedral angle is lower than $90^{\circ}$, the polymer adopts a cis-cisoidal backbone where the internal and external helices rotate in the same direction (Figure $1 b, c$ ). On the other hand, if $\omega_{1}>90^{\circ}$, the polymer adopts a cis-transoidal polyene skeleton where the internal and external helices rotate in opposite directions (Figure 1b,c).

When this structural variability is combined with the complications arisen from the presence of the same repetition fragment-monomer repeating unit-along the polymer chain, a powerful structural technique such as nuclear magnetic resonance is almost useless.

Solid-state methods such as X-ray diffraction ${ }^{8-18}$ or atomic force microscopy (AFM) ${ }^{19-27}$ can be used to provide the

Received: January 14, 2019

Accepted: February 8, 2019

Published: March 13, 2019 
a)

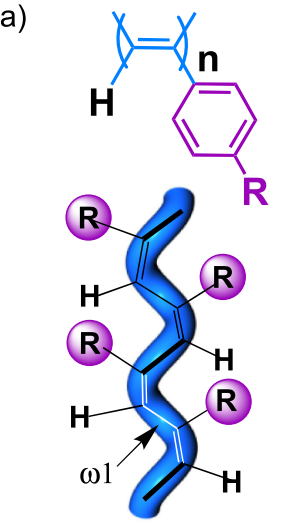

b)

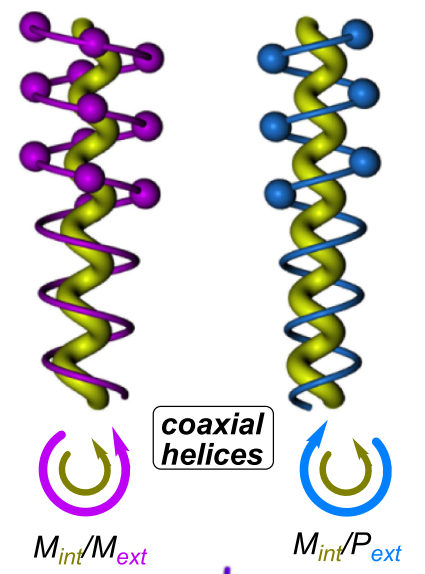

c)

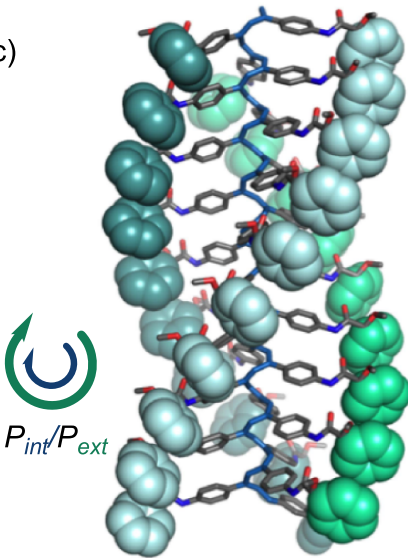

cis-cisoidal

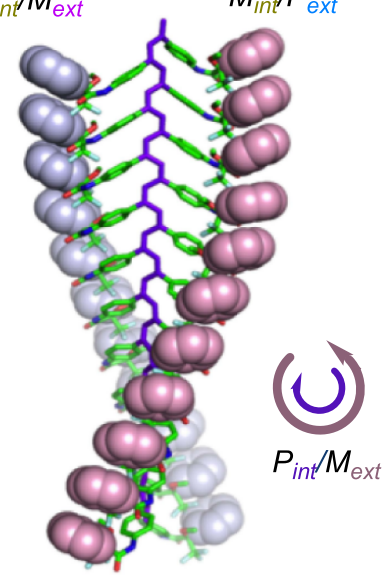

cis-transoidal

Figure 1. (a) Chemical structure of PPAs and its folding into a helix. (b) Schematic representation of different scaffolds formed by two coaxial helices rotating in the same and opposite directions. (c) 3D model of PPAs showing two different scaffolds where the coaxial helices rotate in the same or opposite direction.

helical pitch, length, and helix width, although no information about the internal helical sense is obtained.

AFM can give details on the orientation of the external part of the helix (Figure 2), although high-resolution AFM images are required. ${ }^{19-27}$ These images are obtained from twodimensional crystals or self-assembled monolayers, which cannot always be successfully prepared. ${ }^{27}$ Therefore, the application of this technique to the structural analysis of PPAs is limited.

For its part, differential scanning calorimetry (DSC) is known to provide information about the internal helix of the PPA. Thus, cis-cisoidal $(\mathrm{c}-\mathrm{c})$ and cis-transoidal $(\mathrm{c}-\mathrm{t})$ polyene backbones are associated with different and characteristic thermograms ${ }^{28}$ (Figure 2).

Unfortunately, these studies are frequently limited by the poor definition of the thermograms or by the presence of peaks associated with conformational changes in the pendants, making the assignments unreliable.

Recently, we have shown that vibrational circular dichroism (VCD) can give important information about the orientation of the external part of the PPA helix. ${ }^{29}$ Thus, the solvent can interact with the pendant groups present at the periphery of the PPA forming a supramolecular helix with the same orientation than the external part of the PPA helix. In this way, the VCD sign due to the solvent bands reflects the orientation
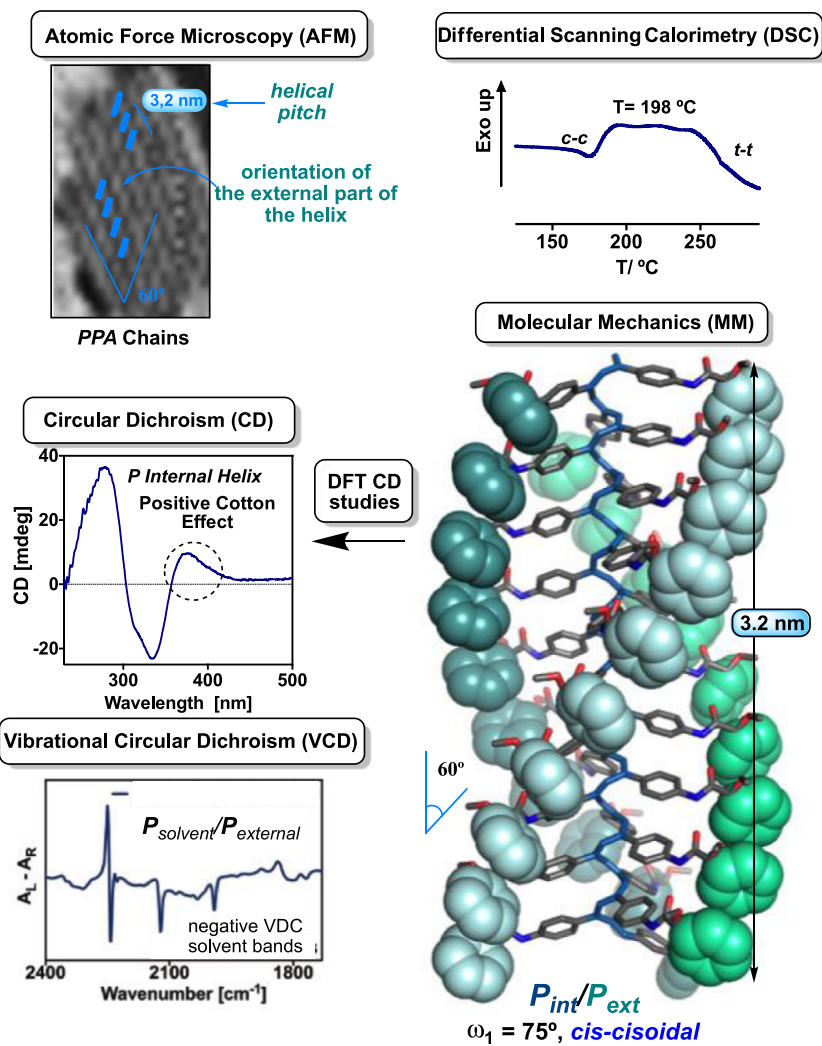

Figure 2. Combination of structural information obtained from a PPA by different structural techniques such as AFM, DSC, molecular mechanics, DFT, ECD, and VCD.

of the external part of the helix (Figure 2) and can be used to determine its sense.

The combination of experimental methods with theoretical calculations has also been used to elucidate the secondary structure of some helical polymers different from PPAs, for instance, through $\mathrm{VCD},{ }^{30-32}$ electronic circular dichroism (ECD) simulations, and MMFF94 ${ }^{33}$ energy minimization.

ECD is one of the most important structural techniques in the study of PPAs. By looking at the ECD spectra of PPAs, it is possible to determine an excess of a single-handed helix into the polymer because of the presence of an ECD signature at the vinylic region (Figure 2). However, no helical parameters can be obtained from these experiments. ${ }^{1-5}$

Lately, we found that it is possible to determine the $\mathrm{P} / \mathrm{M}$ internal helical sense of a PPA from the ECD spectra. This conclusion is based on the correlation observed between a series of PPAs with different and well-known helical scaffolds (Figure 2) and the theoretically calculated time-dependent density functional theory (TD-DFT) ECD spectra. ${ }^{34}$ Thus, a positive Cotton effect at the vinylic region corresponds to a $\mathrm{P}$ helical (internal) sense, whereas a negative Cotton effect indicates the presence of an $\mathrm{M}$ helix for the polyene skeleton.

This correlation works fine in the different PPAs tested, whose secondary structure was obtained from a combination of different structural techniques.

In this paper, we evaluate the robustness of this ECD trace/ internal helical sense relationship, analyzing how variations in the main dihedral angles- $\omega_{1}, \omega_{2}, \omega_{3}, \omega_{4}$-of the PPAs can affect their ECD spectra, and therefore the reliability of the helical sense assignment (Figure 3). 

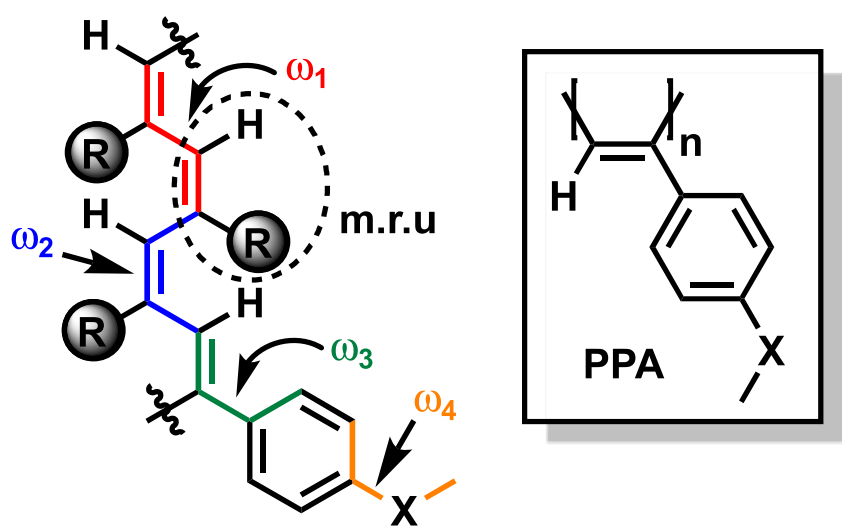

Figure 3. Main dihedral angles of a PPA.

\section{RESULTS AND DISCUSSION}

The helical structure adopted by a PPA is mainly dependent on the values adopted by the $\mathrm{C}=\mathrm{C}-\mathrm{C}=\mathrm{C}\left(\omega_{1}\right)$ and the $\mathrm{C}-\mathrm{C}=$ $\mathrm{C}-\mathrm{C}\left(\omega_{2}\right)$ dihedral angles. All the polymers mentioned in this study (prepared with $\mathrm{Rh}(\mathrm{I})$ as the catalyst) have a cis configuration of the double bonds, and therefore, a value $\omega_{2}=$ $0^{\circ}$ was introduced in all the three-dimensional (3D) models along this work.

Before studying the effect of rotations around $\omega_{1}, \omega_{3}$, and $\omega_{4}$ on the PPA ECD spectra, we checked the minimum size of an oligo(phenylacetylene) [number of monomer repeating units $(n)$ ] required to reach convergence in the ECD shape and therefore for the computational study to reproduce the experimental ECD spectra.

From previous studies, we know that a PPA can adopt two main different scaffolds attending to the value adopted by $\omega_{1}$. Thus, on the one hand, if $\omega_{1}<90^{\circ}$, the polyene adopts a compressed cis-cisoidal helical scaffold with three residues per helical turn (Figure 1c), and with the internal and the external helices rotating in the same direction. On the other hand, if $\omega_{1}$ $>90^{\circ}$, the polymer adopts a stretched helical structure, with two residues per turn and with the internal and external helices rotating in opposite directions (Figure 1c).

Considering the above, a series of oligomers was built by varying the number of monomer repeating units $(n)$ in the two different polyene skeletons, that is, cis-cisoidal and cistransoidal. The dihedral angles used to build the two different scaffolds were obtained from previous experimental and theoretical studies. 6,34

In this way, the series of oligomers with cis-cisoidal $n+3$ arrangements was built with $\omega_{1}=60^{\circ}, \omega_{3}=-36^{\circ}$, and $n=3,6$, 9, 12, and 15 monomers (Figure $4 \mathrm{~b}$ ), whereas for the cistransoidal $n+2$ oligomers, we selected $\omega_{1}=165^{\circ}, \omega_{3}=80^{\circ}$, and $n=2,4,6,8,10,12,14$, and 16 units (Figure 4c).

No substituents were introduced on the phenyl ring of the PPAs, and therefore, $\omega_{4}$ is irrelevant in these calculations.

TD-DFT CAM-B3LYP/6-31G* ECD calculations ${ }^{35-40}$ produce theoretical spectra in oligomers with $n \leq 6$ that do not follow the typical ECD signature of a helical structure with three alternating positive and negative Cotton effects. A good convergence between experimental and theoretically calculated ECD spectra requires the presence of at least nine residues in the oligomer chain $(n=9)$ (Figure $4 \mathrm{~b}, \mathrm{c})$. Thus, in the case of cis-cisoidal oligomers (Figure $4 \mathrm{~b}$ ), the calculated ECD spectrum with $n=6$ does not fit the experimental ECD spectrum, but from $n=9$ and above, the stationary points are

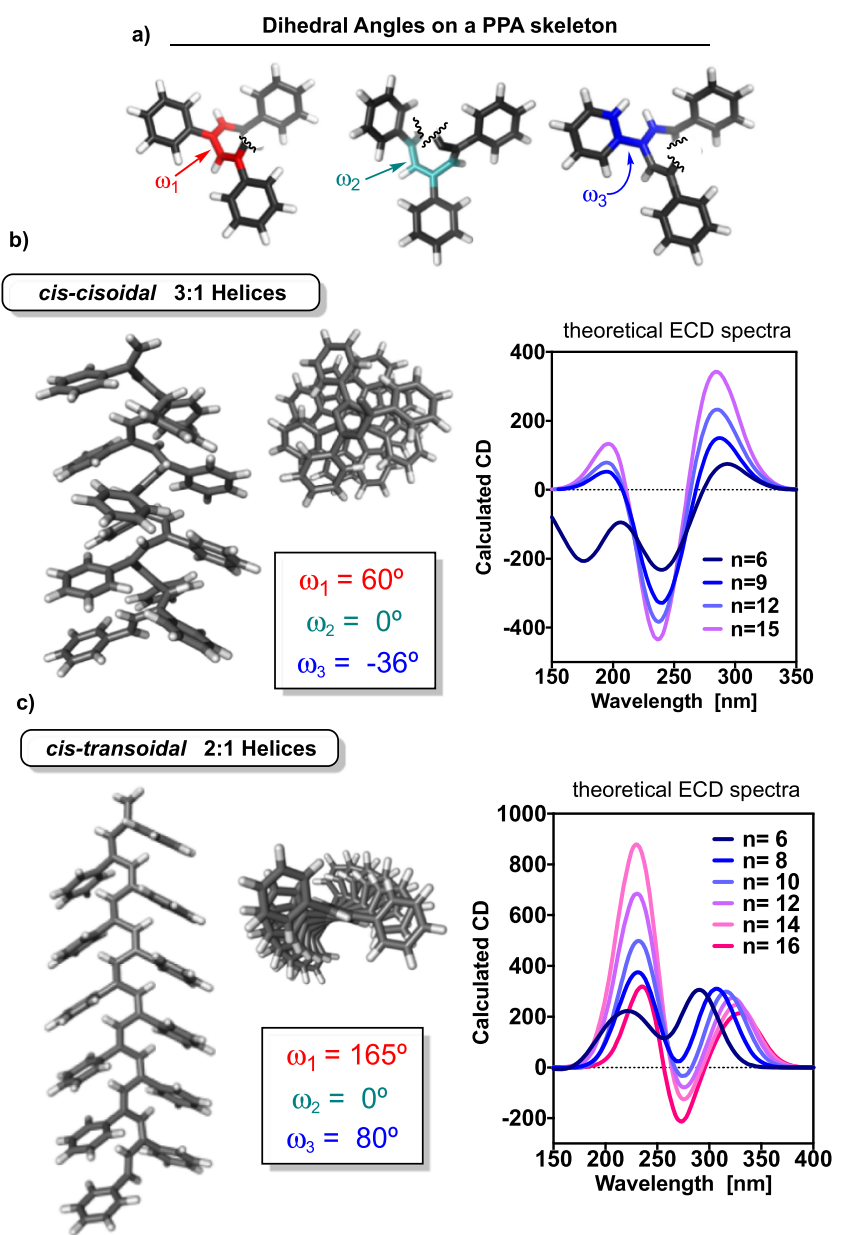

Figure 4. (a) Main dihedral angles of a PPA used in an ECD dependence study. TD-DFT CAM-B3LYP/6-31G* ECD spectra of different size oligomers with (b) a cis-cisoidal and (c) a cistransoidal scaffold. Full width at half-maximum (FWHM) equals 0.62 $\mathrm{eV}$.

in agreement in wavelength and in intensity with an experimental cis-cisoidal ECD spectrum. In PPAs with a cis-transoidal disposition (Figure 2a), convergence in the lambda region of $330 \mathrm{~nm}$ is reached already at $n=8$, although for convergence of the full ECD spectrum, $n \geq 10$ is necessary (Figure 4c).

Taking these results into account, we selected for the theoretical ECD studies oligomers with $n=12$, as good representatives of both $n+2$ and the $n+3$ scaffolds.

Next, we studied how variations in $\omega_{1}$ and $\omega_{3}\left(\omega_{2}=0^{\circ}\right.$ in cis polymers) can affect the ECD signature of a PPA.

Thus, ECD spectra were evaluated for cis-cisoidal 12-mer oligophenylacetylenes with $\omega_{1}=60^{\circ}, 70^{\circ}$, and $80^{\circ}$. Similar calculations were also carried out onto 12 -mer cis-transoidal oligo(phenylacetylene)s with $\omega_{1}=155^{\circ}, 165^{\circ}$, and $175^{\circ}$. All the studies were performed for positive values of $\omega_{1}$, which produced a $\mathrm{P}$ internal helix. $\omega_{2}$ was fixed in all cases at $0^{\circ}$, in accordance with the cis configuration of the double bond.

Variations on $\omega_{3}$ were introduced as negative values (from $0^{\circ}$ to $-90^{\circ}$ ) because most of the reported right-handed ciscisoidal and cis-transoidal helices present a negative value for this dihedral angle. In addition, modeling showed us that negative $\omega_{3}$ angles give the best fits for the right-handed ciscisoidal and cis-transoidal polyene skeletons. 
In some special cases, $\omega_{3}$ could be introduced with a positive value, being this feasible just when $\omega_{1}$ is close to $90^{\circ}$ (i.e. $\omega_{1}=$ $80^{\circ}$, Figure $5 \mathrm{c}$ ), otherwise too unstable high-energy conformations result. In a similar manner, geometries with $\omega_{3}=$ $30^{\circ}$ or larger were not considered because of their high energies.
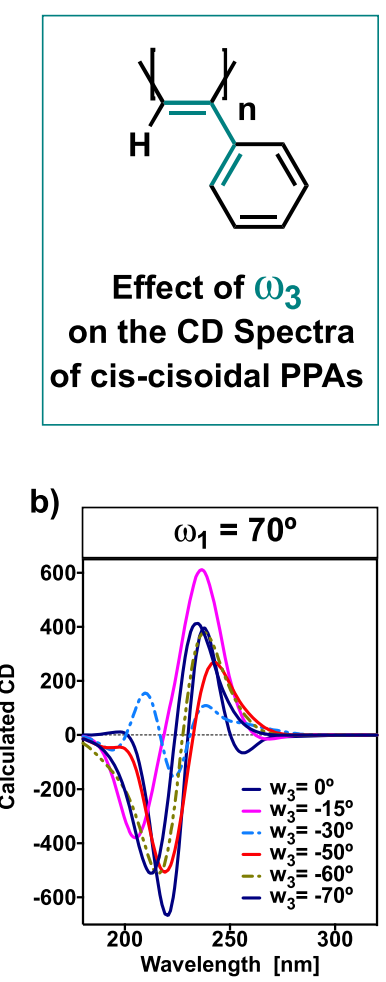

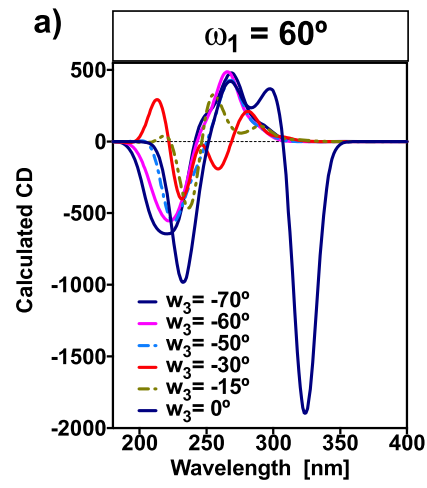

c)

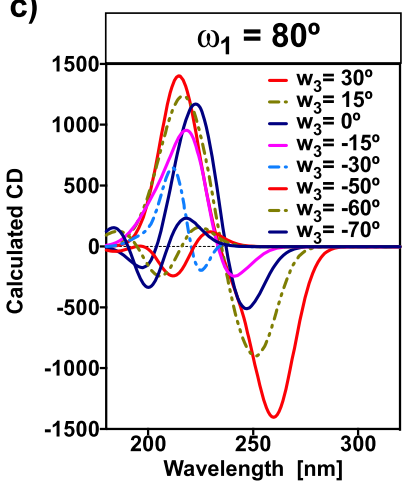

Figure 5. TD-DFT CAM-B3LYP/6-31G* ECD spectra of a 12 -mer oligo(phenylacetylene) with different cis-cisoidal scaffolds: (a) $\omega_{1}=$ $60^{\circ}$, (b) $\omega_{1}=70^{\circ}$, and (c) $\omega_{1}=80^{\circ}$ showing different $\omega_{3}$ values. FWHM equals to $0.276 \mathrm{eV}$.

Finally, the absence of substituents on the phenyl ring of these oligo(phenylacetylene)s makes $\omega_{4}$ irrelevant.

Next, we examined how variations in $\omega_{3}$ values can affect the calculated ECD for several cis-cisoidal structures $\left(\omega_{1}<90^{\circ}\right)$ (Figure 5).

On the one hand, it was found that in those cases where $\omega_{1}$ and $\omega_{3}$ have opposite signs $-\omega_{1}>0^{\circ}$ and $\omega_{3}<0^{\circ}-$, the ECD trace shows two Cotton effect bands, the first positive and the second negative. More precisely, if $\omega_{1}=60^{\circ}$ and $\omega_{3}=-15^{\circ}$, $-30^{\circ},-50^{\circ},-60^{\circ}$, and $-70^{\circ}$, a first positive Cotton effect appears at around $270 \mathrm{~nm}$, whereas a second negative Cotton band is distinguished at $230 \mathrm{~nm}$ (Figure 5a). On the other hand, in those oligomers with $\omega_{1}=70^{\circ}$ and $\omega_{3}=-15^{\circ},-30^{\circ}$, $-50^{\circ},-60^{\circ}$, and $-70^{\circ}$, the two Cotton effect bands appear shifted toward lower wavelengths: the first positive band at 240 $\mathrm{nm}$ and the second negative at $220 \mathrm{~nm}$ (Figure 5b). The same effect is observed in cis-cisoidal structures with $\omega_{1}=80^{\circ}$ and $\omega_{3}=-50^{\circ},-60^{\circ}$, and $-70^{\circ}$, now showing the first, positive, Cotton band at $220 \mathrm{~nm}$ and the second, negative, at $210 \mathrm{~nm}$ (Figure 5c).

Thus, increases in the $\omega_{1}$ angle shift the two Cotton bands toward lower wavelengths, as could be expected from a lower conjugation among the double bonds.

In order to unambiguously determine which one of the dihedral angles, $\omega_{1}$ and/or $\omega_{3}$, is associated with the first positive Cotton effect, the molecular orbitals (MOs) obtained from the theoretical calculations were analyzed. Thus, we can determine which MOs contribute most to the excitations responsible of the first Cotton effect.

In the structures where $\omega_{1}$ and $\omega_{3}$ have opposite signs $-\omega_{1}$ $>0^{\circ}$ and $\omega_{3}<0^{\circ}-$, the calculations showed that the main excitations contributing to the first Cotton effect are due to MOs on the phenyl ring and on the polyene backbone. These excitations are closely related to $\omega_{1}$ and $\omega_{3}$, which induce in both cases a positive Cotton effect. Therefore, this first positive Cotton effect can be correlated with a right-handed orientation for the polyene backbone (Figure 6a and Supporting Information).

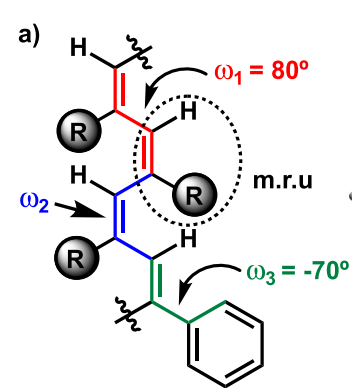

b)

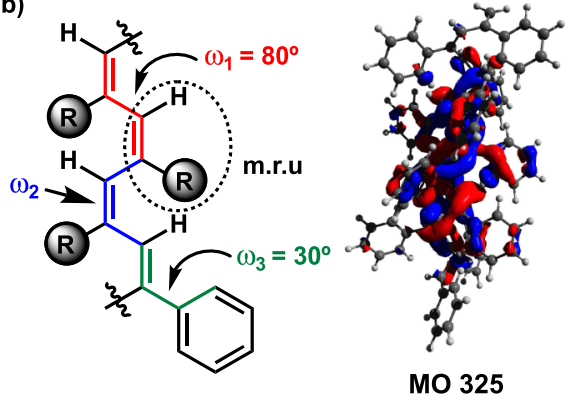

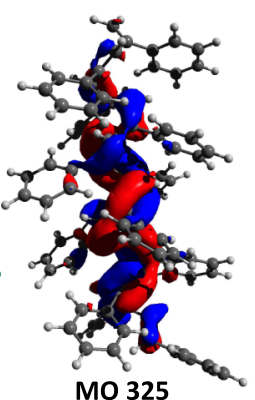
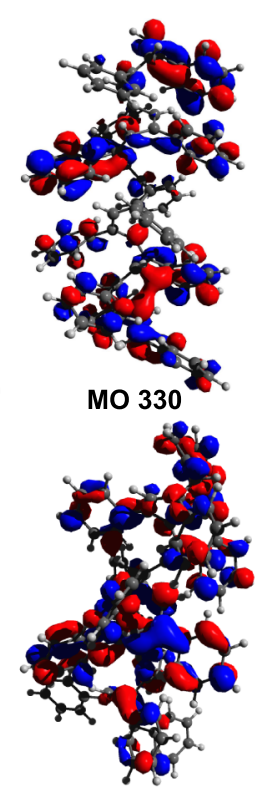

MO 333
Figure 6. MOs responsible for the first Cotton effect in two different calculated oligo(phenylacetylene)s. (a) cis-cisoidal PPA $\omega_{1}=80^{\circ}$ and $\omega_{3}=-70^{\circ}$. (b) cis-cisoidal PPA $\omega_{1}=80^{\circ}$ and $\omega_{3}=30^{\circ}$. FWHM equals to $0.276 \mathrm{eV}$.

Interestingly, when $\omega_{3}$ is zero $\left(\omega_{1}=60^{\circ}, 70^{\circ}\right.$, and $\left.80^{\circ}\right)$, or when $\omega_{1}$ and $\omega_{3}$ have the same positive sign $-\omega_{1}=80^{\circ} / \omega_{3}=$ $15^{\circ}$ and $30^{\circ}$-, an extra Cotton effect appears at higher wavelengths-around $330 \mathrm{~nm}$ for $\omega_{1}=60^{\circ}$ (Figure 5a), 260 $\mathrm{nm}$ for $\omega_{1}=70^{\circ}$ (Figure 5b), and 250-260 nm for $\omega_{1}=80^{\circ}$ (Figure 5c)-, whereas the other two Cotton effects remain either unaffected $\left(\omega_{1}=60^{\circ}\right.$ and $70^{\circ}$, Figure 5a,b) or converge into a single positive Cotton effect at around $230 \mathrm{~nm}$ (Figure $5 c)$.

Analysis of the MOs involved in these theoretical ECD spectra shows that when $\omega_{1}>0$ and $\omega_{3}>0$, the MOs on the phenyl ring and on the polyene backbone do not contribute to the first Cotton effect in the same manner as shown for ciscisoidal PPAs with opposite signs for $\omega_{1}$ and $\omega_{3}$ (Figure 6 and Supporting Information).

Transitions among these MOs generate now two different Cotton effects with opposite signs, as the excitations involved in the first Cotton effect in the case of opposite $\omega_{1}$ and $\omega_{3}$ signs are now divided into two different Cotton effects (Figure 5 ). The second Cotton effect resonates at the same frequency than the first Cotton effect in polymers where $\omega_{1}$ and $\omega_{3}$ have opposite signs. This Cotton effect is positive in both cases and 
seems to indicate a right-handed helix for the polyene backbone. The new first Cotton effect appears at higher wavelengths and has opposite sign.

Thus, from these studies, we found that in cis-cisoidal PPAs, the first Cotton effect seems to be directly associated with a right- or left-handed orientation of the polyene scaffold $\left(\omega_{1}\right)$. In those special cases where $\omega_{1}$ and $\omega_{3}$ have equal signs or $\omega_{3}=0^{\circ}$, an extra Cotton effect should appear, shifted toward larger wavelengths, and to determine the internal helical sense of the polymer, it is necessary to take into account the sign of the second Cotton effect.

Next, we carried out the same study on the cis-transoidal oligomer skeletons $\left(\omega_{1}>90^{\circ}\right)$. In this backbone, $\omega_{3}$ and $\omega_{1}$ have to be introduced with opposite signs. Otherwise, very constrained helical structures are generated, which are not worthwhile considering.

Thus, ECD calculations on cis - transoidal $n=12$ oligomers with $\omega_{1}=155^{\circ}, 165^{\circ}$, and $175^{\circ}$ and $\omega_{3}$ varying from $-30^{\circ}$ to $-90^{\circ}$ showed in all cases a first positive Cotton effect, which resonates at different wavelengths because of the different conjugation of double bonds (Figure 7). During these studies, we found that although modulation of $\omega_{3}$ produces some changes in the overall ECD spectra, the first Cotton effect remains positive in all cases (Figure 7 ).

This first Cotton effect is due to contributions from MOs on the polyene skeleton $\left(\omega_{1}\right)$ (Figure $7 \mathrm{~d}$ ), and therefore, in cistransoidal PPAs, there is a correlation between the sign of the first Cotton effect and the helical sense of the internal helixECD-positive/P helix and ECD-negative/M helix.

Finally, in order to get a more complete vision on the structural factors influencing the ECD of a PPA, we decided to check what could be the contribution to the ECD trace of a substituent on the phenyl ring.

Many PPAs with amide function on the phenyl ring have been prepared and have their ECDs reported in the literature. Therefore, we decided to study the influence on the ECD of $\omega_{4}$ rotations on para-formamido PPA oligomers (Figure 8a). More precisely, we built para-formamido oligo(phenylacetylene)s $n=12$, with two different cis-cisoidal backbones- $\omega_{1}=60^{\circ}, \omega_{3}=-60^{\circ}$, and $\omega_{1}=70^{\circ}, \omega_{3}=-30^{\circ}-$ and with one cis-transoidal $-\omega_{1}=165^{\circ}, \omega_{3}=-75^{\circ}$ (Figure $8 \mathrm{a}$.

The ECD was calculated considering that the amide group can adopt different orientations depending on the $\omega_{4}$ that was introduced in a range from $10^{\circ}$ to $135^{\circ}$. As a result, we observed that changing $\omega_{4}$, in both cis-cisoidal and cistransoidal para-formamido oligophenylacetylenes, does not affect the sign of the first Cotton band that remains positive for all rotamers. Therefore, in amide-substituted PPAs, the correlation between the sign of the first Cotton band and the helical sense of the internal helix-positive first Cotton effect/P helix-conserves its predictive value. Only the bands at lower wavelengths of the ECD spectra are affected by this dihedral angle $\left(\omega_{4}\right)$.

Thus, from these studies, we can assert that $\omega_{1}$ and $\omega_{3}$ are the dihedral angles involved in the formation of the first Cotton effect of a PPA ECD trace. Thus, by looking at its sign-positive or negative-it is possible to determine the helical sense of the PPA, although first it is necessary to discriminate between those polymers that present an ECD trace with three or four alternating Cotton effects.

In the literature, we found that the most frequent situation is a PPA presenting an ECD trace with three alternating Cotton
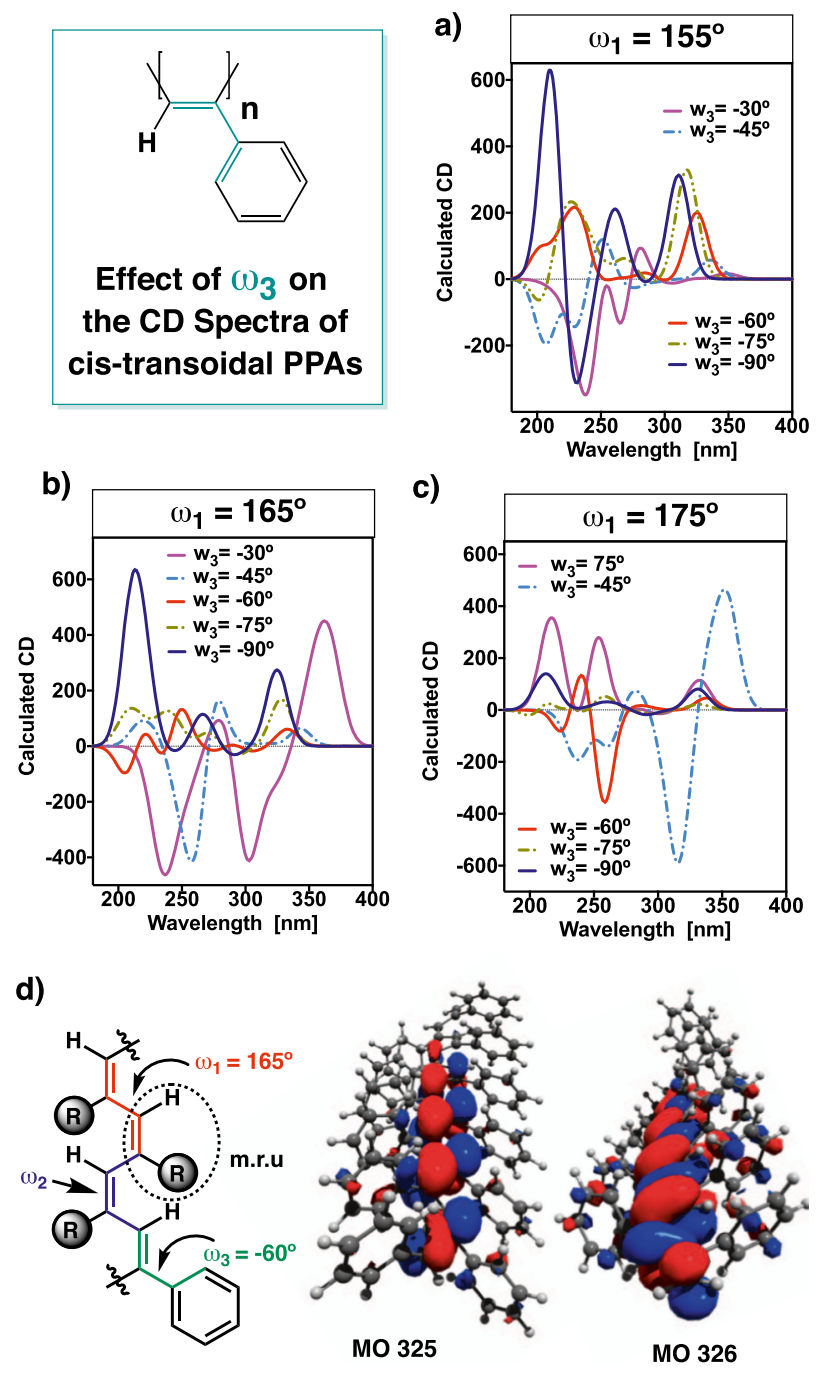

Figure 7. TD-DFT CAM-B3LYP/6-31G* ECD spectra of a 12-mer oligophenylacetylene with different cis-transoidal scaffolds: (a) $\omega_{1}=$ $155^{\circ}$, (b) $\omega_{1}=165^{\circ}$, and (c) $\omega_{1}=175^{\circ}$ showing different $\omega_{3}$ values. (d) MOs involved in the sign of the first Cotton effect. FWHM equals to $0.276 \mathrm{eV}$.

effects. ${ }^{6,7,27,29,34}$ In this case, correlation between the sign of the first Cotton band and the helical sense of the internal helix is direct-positive first Cotton effect/P helix or negative first Cotton effect/M helix. Sometimes, PPAs show four alternating Cotton effects in their ECD trace, which makes the helical sense assignment difficult. Herein, we could identify the position-first Cotton effect-and the dihedral angle responsible of this extra band- $\omega_{3}$. Therefore, in this case, the first Cotton effect does not provide information about the internal helical sense of the polymer. In such cases, the second Cotton effect is the one related to $\omega_{1}$ and to the internal helical sense of the PPA-positive second Cotton effect/P helix or negative second Cotton effect/M helix.

Additionally, in the literature, it is possible to find examples where a cis-cisoidal or cis-transoidal PPA can show an ECD with three or four alternating Cotton effects depending on the solvent used to dissolve the polymer. For instance, a PPA bearing as pendant the Mosher reagent linked as amide on the phenyl ring shows a typical ECD signature, with three alternating positive/negative/positive (or vice versa) Cotton effects when dissolved in chloroform, while an extra band at 

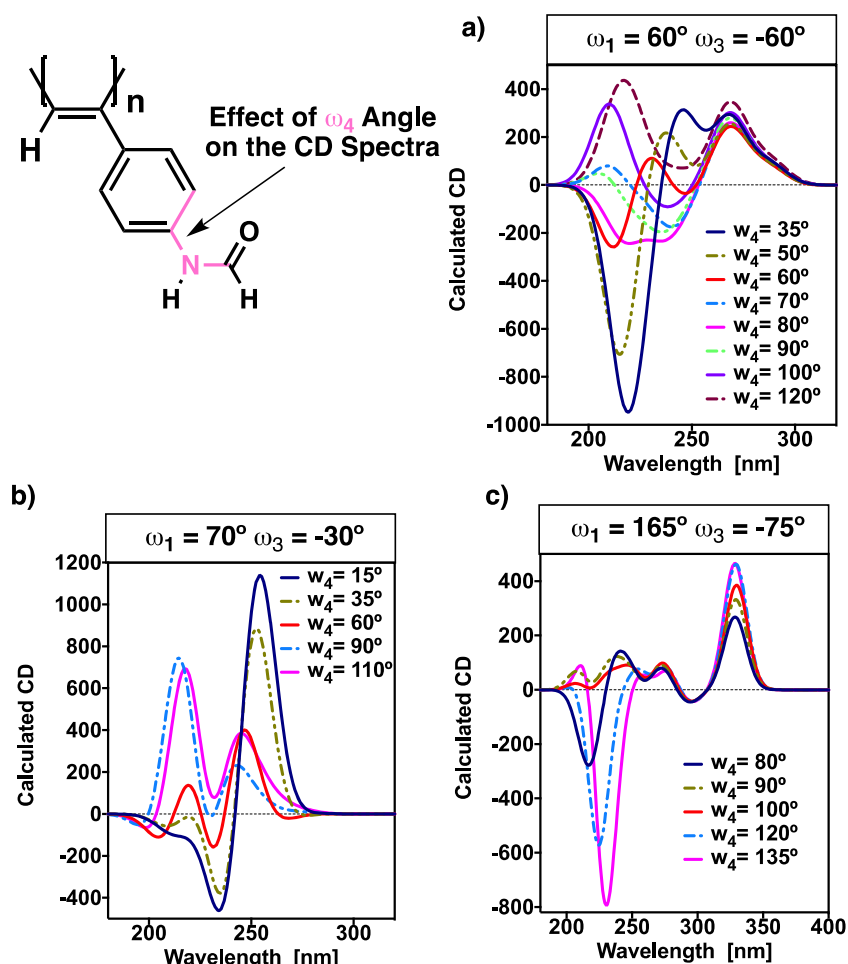

Figure 8. TD-DFT CAM-B3LYP/6-31G* ECD spectra of a 12-mer oligophenylacetylene with different cis-cisoidal (a) $\omega_{1}=60^{\circ}, \omega_{3}=$ $-60^{\circ}$, (b) $\omega_{1}=70^{\circ}, \omega_{3}=-30^{\circ}$ and cis-transoidal (c) $\omega_{1}=165^{\circ}, \omega_{3}$ $=-75^{\circ}$ showing different $\omega_{4}$ values. FWHM equals to $0.276 \mathrm{eV}$.

higher wavelength is observed in tetrahydrofuran (THF) or dimethylformamide (for the ECD in THF see Figure 9a). ${ }^{3}$ In this case, the second Cotton effect at around $380 \mathrm{~nm}$ is the one that indicates the helical sense of the internal helix. ${ }^{7}$ A similar situation is found in PPAs bearing amino acid groups as pendants $^{41}$ (Figure 9b).

Interestingly, we found another example that reports how changes in temperature rotate the $\omega_{3}$ angle producing an inversion of the extra band, while the rest of the ECD spectrum, and therefore the internal helical sense of the PPA, remains unaltered (Figure 9c) $\left(P_{\text {int }}, \operatorname{ECD}(+)\right.$ at $\left.380 \mathrm{~nm}\right) .{ }^{42}$

Overall, this extra band serves to illustrate how the action of external stimuli (changes in polarity/donor ability of the solvent or in temperature) could affect the rotation involved in $\omega_{3}$ that is reflected in the ECD spectrum.

\section{CONCLUSIONS}

In conclusion, TD-DFT calculations for different cis-cisoidal and cis-transoidal oligo(phenylacetylene)s allowed us to demonstrate how the main dihedral angles of a PPA contribute to the different Cotton effects of its ECD spectrum.

Thus, in cis-cisoidal PPAs, the first Cotton effect is produced by excitations involving MOs placed both at the polyene and at the phenyl rings. As a result, two different ECD spectra can be obtained:

1. A typical helical ECD pattern with three alternating Cotton effects-positive/negative/positive or vice versa. In this case, the first Cotton indicates the helical sense of the polyene-that is, ECD-positive/P helix; ECDnegative/M helix (Figure 9d).

2. An ECD signature with four alternating Cotton effects. In this case, the first and second Cotton effects are
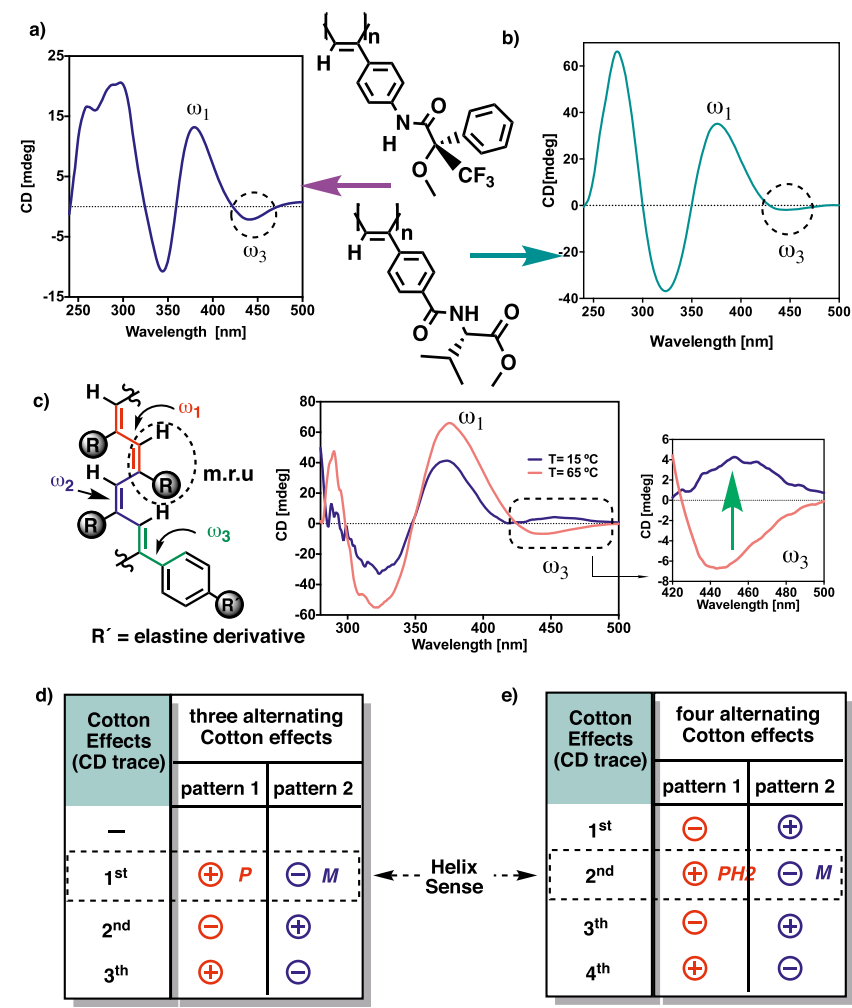

Figure 9. $(\mathrm{a}-\mathrm{c})$ Literature examples of PPAs showing an extra Cotton effect associated with the relative orientation of the $\omega_{1} / \omega_{3}$ dihedral angles. (d) Prediction of the M or P helical sense based on the sign of the first Cotton effect for both cis-cisoidal and cis-transoidal PPAs whose ECDs show three Cotton effects. (e) Prediction of the M or P helical sense based on the sign of the second Cotton effect for both cis-cisoidal and cis-transoidal PPAs whose ECDs show four Cotton effects.

produced by transitions involving MOs on the polyene and on the phenyl rings, being possible to extract the helical sense of the internal helix by using the sign of the second Cotton effect-that is, ECD-positive/P helix; ECD-negative/M helix. The new first Cotton effect contains information about the $\omega_{1} / \omega_{3}$ relationship and has no value for helical sense determination (Figure 9e).

Regarding cis-transoidal PPAs, there is a helical ECD pattern with three alternating Cotton effects. The first Cotton effect of the ECD spectra is mainly generated by contributions of MOs located on the polyene backbone and can be positive or negative depending on the helical sense described by $\omega_{1}$. Therefore, there is a correlation between the sign of the first Cotton effect and the $\mathrm{P} / \mathrm{M}$ helical sense described by the internal helix-that is, ECD-positive/P helix; ECD-negative/ $\mathrm{M}$ helix. Experimentally, we found that, in some cis-transoidal PPAs, an uncommon ECD trace with four alternating Cotton effects is observed, and in that case-similarly to cis-cisoidal PPAs - the second Cotton effect is the one that contains the information of the internal helix.

Our results were experimentally validated using PPA ECD spectra reported in the literature, where an extra Cotton effect can be observed depending on the solvent used to dissolve the polymer, or even where an inversion of this extra Cotton effect can be produced by temperature changes without affecting the helical sense of the PPA. ${ }^{7,41}$ 
Thus, this is the first time that a PPA ECD signature is broken down, analyzing how the main dihedral angles contribute to the final ECD spectrum, and therefore making it possible to determine the polymer helical sense, in both ciscisoidal and cis-transoidal arrangements, depending on the sign of the first or second Cotton effect in an ECD trace with three or four alternating Cotton effects, respectively.

\section{COMPUTATIONAL DETAILS}

To obtain the ECD spectra using computational chemistry, scalar rotational strengths have to be evaluated. For a transition from the ground state of the molecule (0) to an excited state $(n)$, the scalar rotational strength can be calculated from the residue of the linear response function corresponding to the electric dipole-magnetic dipole polarizability tensor $\left(R_{0 n}^{\mathrm{LG}}\right)$. In the length-gauge (LG) formalism, the residue is given by eq 1 :

$$
R_{0 n}^{\mathrm{LG}}=\sum_{\alpha} \lim _{\omega \rightarrow \omega_{0 n}}\left(\omega-\omega_{0 n}\right) \mu_{\alpha} ; m_{\alpha \omega}
$$

where $\omega_{0 n}$ is the frequency of the transition between the 0 and the $n$ state, $\mu_{\alpha}$ is the $\alpha$ component of the electric dipole moment, and $m_{\alpha}$ is the $\alpha$ component of the magnetic dipole moment of the molecule. ${ }^{35}$

The theoretical ECD spectra are calculated using TDDFT $^{35,36}$ in combination with the CAM-B3LYP ${ }^{37,38}$ density functional and the $6-31 G^{*}$ basis set. ${ }^{39}$ The calculations were carried out with the ORCA program and 80 excitation energies were evaluated. ${ }^{40}$

The TD-DFT CAM-B3LYP/6-31G* ECD plots are built from the calculated rotational strengths using a Gaussian band shape and FWHM corrected to reproduce the shape of the experimental PPA spectra.

\section{ASSOCIATED CONTENT}

\section{(S Supporting Information}

The Supporting Information is available free of charge on the ACS Publications website at DOI: 10.1021/acsomega.9b00122.

Computational details (PDF)

\section{AUTHOR INFORMATION}

\section{Corresponding Authors}

*E-mail: berta.fernandez@usc.es (B.F.).

*E-mail: felix.freire@usc.es (F.F.).

\section{ORCID $\odot$}

Berta Fernández: 0000-0001-6686-6534

Rafael Rodríguez: 0000-0002-8588-7776

Félix Freire: 0000-0002-2672-5830

\section{Author Contributions}

The manuscript was written through contributions from all authors. All authors have given approval to the final version of the manuscript.

\section{Notes}

The authors declare no competing financial interest.

\section{ACKNOWLEDGMENTS}

The financial support from MINECO (CTQ2014-61470-EXP and CTQ2015-70519-P), Xunta de Galicia (GRC2014/040 and ED431C 2017/17, Centro Singular de Investigación de Galicia Accreditation 2016-2019, ED431G/09), the COST Action CM1405 Molecules in Motion (MOLIM), and the
European Regional Development Fund (ERDF) is gratefully acknowledged. We also thank Centro de Supercomputación de Galicia (CESGA) for computational resources. R.R. is grateful to MINECO for an FPI predoctoral fellowship.

\section{ABBREVIATIONS}

PPA, poly(phenylacetylene); ECD, electronic circular dichroism; DFT, density functional theory; TD-DFT, time-dependent density functional theory; B3LYP, Becke three-parameter Lee-Yang-Parr; CAM-B3LYP, Coulomb-attenuated method B3LYP; MM, molecular mechanics; MMFF94, molecular mechanics Merck molecular force field; FWHM, full width at half-maximum; DSC, differential scanning calorimetry; AFM, atomic force microscopy; VCD, vibrational circular dichroism; $\mathrm{XRD}, \mathrm{X}$-ray diffraction; NMR, nuclear magnetic resonance; $\mathrm{MO}$, molecular orbital

\section{REFERENCES}

(1) Freire, F.; Quiñoá, E.; Riguera, R. Chiral nanostructure in polymers under different deposition conditions observed using atomic force microscopy of monolayers: poly(phenylacetylene)s as a case study. Chem. Commun. 2017, 53, 481-492.

(2) Freire, F.; Quiñoá, E.; Riguera, R. Supramolecular Assemblies from Poly(phenylacetylene)s. Chem. Rev. 2016, 116, 1242-1271.

(3) Yashima, E.; Ousaka, N.; Taura, D.; Shimomura, K.; Ikai, T.; Maeda, K. Supramolecular Helical Systems: Helical Assemblies of Small Molecules, Foldamers, and Polymers with Chiral Amplification and Their Functions. Chem. Rev. 2016, 116, 13752-13990.

(4) Yashima, E.; Maeda, K.; Iida, H.; Furusho, Y.; Nagai, K. Helical Polymers: Synthesis, Structures, and Functions. Chem. Rev. 2009, 109, 6102-6211.

(5) Liu, J.; Lam, J. W. Y.; Tang, B. Z. Acetylenic Polymers: Syntheses, Structures, and Functions. Chem. Rev. 2009, 109, 57995867.

(6) Rodríguez, R.; Quiñoá, E.; Riguera, R.; Freire, F. Architecture of Chiral Poly(phenylacetylene)s: From Compressed/Highly Dynamic to Stretched/Quasi-Static Helices. J. Am. Chem. Soc. 2016, 138, 9620-9628.

(7) Leiras, S.; Freire, F.; Seco, J. M.; Quiñoá, E.; Riguera, R. Controlled modulation of the helical sense and the elongation of poly(phenylacetylene)s by polar and donor effects. Chem. Sci. 2013, 4, $2735-2743$.

(8) Nagai, K.; Sakajiri, K.; Maeda, K.; Okoshi, K.; Sato, T.; Yashima, E. Hierarchical Amplification of Macromolecular Helicity in a Lyotropic Liquid Crystalline Charged Poly(phenylacetylene) by Nonracemic Dopants in Water and Its Helical Structure. Macromolecules 2006, 39, 5371-5380.

(9) Morimoto, M.; Tamura, K.; Nagai, K.; Yashima, E. Chirality Sensing of Chiral Pyrrolidines and Piperazines with a Liquid Crystalline Dynamic Helical Poly(phenylacetylene) Bearing Ethyl Phosphonate Pendant Groups. J. Polym. Sci., Part A: Polym. Chem. 2010, 48, 1383-1390.

(10) Fukushima, T.; Kimura, H.; Tsuchihara, K. Color and Chiroptical Control of Poly(phenylacetylene) Films with Chiral Hydroxyl Group. Macromolecules 2009, 42, 8619-8626.

(11) Okoshi, K.; Kajitani, T.; Nagai, K.; Yashima, E. Uniaxial Orientation of a Rodlike Helical Poly(phenylacetylene) in an Electric Field. Macromolecules 2008, 41, 258-261.

(12) Mawatari, Y.; Tabata, M.; Sone, T.; Ito, K.; Sadahiro, Y. Origin of Color of $\pi$-Conjugated Columnar Polymers. 1. Poly(p-3methylbutoxy)phenylacetylene Prepared Using a [ Rh(norbornadiene) $\mathrm{Cl}]_{2}$ Catalyst. Macromolecules 2001, 34, 3776-3782.

(13) Motoshige, A.; Mawatari, Y.; Motoshige, R.; Yoshida, Y.; Tabata, M. Contracted helix to stretched helix Rearrangement of an aromatic polyacetylene prepared inn-hexane with [Rh(norbornadiene) $\mathrm{Cl}]_{2}$-triethylamine catalyst. J. Polym. Sci., Part A: Polym. Chem. 2013, 51, 5177-5183. 
(14) Motoshige, R.; Mawatari, Y.; Motoshige, A.; Yoshida, Y.; Sasaki, T.; Yoshimizu, H.; Suzuki, T.; Tsujita, Y.; Tabata, M. Mutual Conversion Between Stretched and Contracted Helices Accompanied by a Drastic Change in Color and Spatial Structure of Poly(phenylacetylene) Prepared with a $[\mathrm{Rh}(\mathrm{nbd}) \mathrm{Cl}]_{2}$-amine Catalyst. J. Polym. Sci., Part A: Polym. Chem. 2014, 52, 752-759.

(15) Percec, V.; Rudick, J. G.; Peterca, M.; Wagner, M.; Obata, M.; Mitchell, C. M.; Cho, W.-D.; Balagurusamy, V. S. K.; Heiney, P. A. Thermoreversible Cis-Cisoidal to Cis-Transoidal Isomerization of Helical Dendronized Polyphenylacetylenes. J. Am. Chem. Soc. 2005, 127, 15257-15264.

(16) Percec, V.; Peterca, M.; Rudick, J. G.; Aqad, E.; Imam, M. R.; Heiney, P. A. Self-Assembling Phenylpropyl Ether Dendronized Helical Polyphenylacetylenes. Chem.-Eur. J. 2007, 13, 9572-9581.

(17) Percec, V.; Aqad, E.; Peterca, M.; Rudick, J. G.; Lemon, L.; Ronda, J. C.; De, B. B.; Heiney, P. A.; Meijer, E. W. Steric Communication of Chiral Information Observed in Dendronized Polyacetylenes. J. Am. Chem. Soc. 2006, 128, 16365-16372.

(18) Percec, V.; Rudick, J. G.; Peterca, M.; Aqad, E.; Imam, M. R.; Heiney, P. A. Synthesis, Structural, and Retrostructural Analysis of Helical Dendronized Poly(1-naphthylacetylene)s. J. Polym. Sci., Part A: Polym. Chem. 2007, 45, 4974-4987.

(19) Sakurai, S.-i.; Okoshi, K.; Kumaki, J.; Yashima, E. TwoDimensional Surface Chirality Control by Solvent-Induced Helicity Inversion of a Helical Polyacetylene on Graphite. J. Am. Chem. Soc. 2006, 128, 5650-5651.

(20) Sakurai, S.-i.; Okoshi, K.; Kumaki, J.; Yashima, E. TwoDimensional Hierarchical Self-Assembly of One-Handed Helical Polymers on Graphite. Angew. Chem., Int. Ed. 2006, 45, 1245-1248.

(21) Nishimura, T.; Takatani, K.; Sakurai, S.-i.; Maeda, K.; Yashima, E. A Helical Array of Pendant Fullerenes on an Optically Active Polyphenylacetylene. Angew. Chem., Int. Ed. 2002, 41, 3602-3604.

(22) Sakurai, S.-i.; Ohira, A.; Suzuki, Y.; Fujito, R.; Nishimura, T.; Kunitake, M.; Yashima, E. Synthesis and Property of Helical poly(phenylacetylene)s Bearing Chiral Ruthenium Complexes and Real Space Imaging of Meso- and Nanoscopic Structures. J. Polym. Sci., Part A: Polym. Chem. 2004, 42, 4621-4640.

(23) Okoshi, K.; Sakurai, S.-i.; Ohsawa, S.; Kumaki, J.; Yashima, E. Control of Main-Chain Stiffness of a Helical Poly(phenylacetylene) by Switching On and Off the Intramolecular Hydrogen Bonding through Macromolecular Helicity Inversion. Angew. Chem., Int. Ed. 2006, 45, 8173-8176.

(24) Sakurai, S.-i.; Ohsawa, S.; Nagai, K.; Okoshi, K.; Kumaki, J.; Yashima, E. Two-Dimensional Helix-Bundle Formation of a Dynamic Helical Poly(phenylacetylene) with Achiral Pendant Groups on Graphite. Angew. Chem., Int. Ed. 2007, 46, 7605-7608.

(25) Ohsawa, S.; Sakurai, S.-i.; Nagai, K.; Banno, M.; Maeda, K.; Kumaki, J.; Yashima, E. Hierarchical Amplification of Macromolecular Helicity of Dynamic Helical Poly(phenylacetylene)s Composed of Chiral and Achiral Phenylacetylenes in Dilute Solution, Liquid Crystal, and Two-Dimensional Crystal. J. Am. Chem. Soc. 2011, 133, $108-114$.

(26) Ohsawa, S.; Sakurai, S.-i.; Nagai, K.; Maeda, K.; Kumaki, J.; Yashima, E. Amplification of macromolecular helicity of dynamic helical poly(phenylacetylene)s bearing non-racemic alanine pendants in dilute solution, liquid crystal and two-dimensional crystal. Polym. J. 2012, 44, 42-50.

(27) Rodríguez, R.; Arias, S.; Quiñoá, E.; Riguera, R.; Freire, F. The role of the secondary structure of helical poly(phenylacetylene)s in the formation of nanoparticles from polymer-metal complexes (HPMCs). Nanoscale 2017, 9, 17752-17757.

(28) Liu, L.; Namikoshi, T.; Zang, Y.; Aoki, T.; Hadano, S.; Abe, Y.; Wasuzu, I.; Tsutsuba, T.; Teraguchi, M.; Kaneko, T. Top-Down Preparation of Self-Supporting Supramolecular Polymeric Membranes Using Highly Selective Photocyclic Aromatization of Cis-Cisoid Helical Poly(phenylacetylene)s in the Membrane State. J. Am. Chem. Soc. 2013, 135, 602-605.

(29) Nieto-Ortega, B.; Rodríguez, R.; Medina, S.; Quiñoá, E.; Riguera, R.; Casado, J.; Freire, F.; Ramírez, F. J. Sequential Induction of Chirality in Helical Polymers: From the Stereocenter to the Achiral Solvent. J. Phys. Chem. Lett. 2018, 9, 2266-2270.

(30) Hase, Y.; Nagai, K.; Iida, H.; Maeda, K.; Ochi, N.; Sawabe, K.; Sakajiri, K.; Okoshi, K.; Yashima, E. Mechanism of helix induction in poly(4-carboxyphenyl isocyanide) with chiral amines and memory of the macromolecular helicity and its helical structures. J. Am. Chem. Soc. 2009, 131, 10719-10732.

(31) Kawauchi, T.; Kumaki, J.; Kitaura, A.; Okoshi, K.; Kusanagi, H.; Kobayashi, K.; Sugai, T.; Shinohara, H.; Yashima, E. Encapsulation of fullerenes in a helical PMMA cavity leading to a robust processable complex with a macromolecular helicity memory. Angew. Chem., Int. Ed. 2008, 47, 515-519.

(32) Tang, H.-Z.; Novak, B. M.; He, J.; Polavarapu, P. L. A thermal and solvocontrollable cylindrical nanoshutter based on a single screwsense helical polyguanidine. Angew. Chem., Int. Ed. 2005, 44, 72987301.

(33) Suzuki, Y.; Tabei, J.; Shiotsuki, M.; Inai, Y.; Sanda, F.; Masuda, T. Synthesis and Helical Structure of Poly(N-butynylamide)s Having Various Side Chains, Where the Helix Is Highly Affected by the Methyl Branch and the Lactone Moiety. Macromolecules 2008, 41, 1086-1093.

(34) Fernández, B.; Rodríguez, R.; Rizzo, A.; Quiñoá, E.; Riguera, R.; Freire, F. Predicting the Helical Sense of Poly(phenylacetylene)s from their Electron Circular Dichroism Spectra. Angew. Chem., Int. Ed. 2018, 57, 3666.

(35) Rizzo, A.; Coriani, S.; Ruud, K. Computational Strategies for Spectroscopy; From Small Molecules to Nano Systems; Barone, V., Ed.; John Wiley \& Sons: New Jersey, 2012; p 77.

(36) Runge, E.; Gross, E. K. U. Density-Functional Theory for Time-Dependent Systems. Phys. Rev. Lett. 1984, 52, 997-1000.

(37) Yanai, T.; Tew, D. P.; Handy, N. C. A new hybrid exchangecorrelation functional using the Coulomb-attenuating method (CAMB3LYP). Chem. Phys. Lett. 2004, 393, 51-57.

(38) Peach, M. J. G.; Helgaker, T.; Sałek, P.; Keal, T. W.; Lutnæs, O. B.; Tozer, D. J.; Handy, N. C. Assessment of a Coulomb-attenuated exchange-correlation energy functional. Phys. Chem. Chem. Phys. 2006, 8, 558-562.

(39) Ditchfield, R.; Hehre, W. J.; Pople, J. A. Self-Consistent Molecular-Orbital Methods. IX. An Extended Gaussian-Type Basis for Molecular-Orbital Studies of Organic Molecules. J. Chem. Phys. 1971, $54,724-728$.

(40) Neese, F. The ORCA program system. Wiley Interdiscip. Rev.: Comput. Mol. Sci. 2012, 2, 73.

(41) Lai, L. M.; Lam, J. W. Y.; Tang, B. Z. Synthesis and chiroptical properties of L-valine-containing poly(phenylacetylene)s with (a)chiral pendant terminal groups. J. Polym. Sci., Part A: Polym. Chem. 2006, 44, 2117-2129.

(42) Arias, S.; Freire, F.; Calderón, M.; Bergueiro, J. Unexpected Chiro-Thermoresponsive Behavior of Helical Poly(phenylacetylene)s Bearing Elastin-Based Side Chains. Angew. Chem., Int. Ed. 2017, 56, 11420-11425. 